
Does facial expressivity count? How typically developing children respond initially to children with autism. 


\begin{abstract}
Research investigating expressivity in children with autism spectrum disorder (ASD) has reported flat affect or bizarre facial expressivity within this population. The impact expressivity may have on first impression formation has received little research input. We examined how videos of children with ASD were rated for expressivity by adults blind to the condition. We further investigated the friendship ratings given by 44 typically developing (TD) children to the same videos. These ratings were compared to friendship ratings given to video clips of typically developing children. Results demonstrated that adult raters, blind to the diagnosis of the children in the videos, rated ASD children as being less facially expressive than TD children. These ASD children in the videos were also rated lower on all aspects of our friendship measures when compared with the TD children. Results suggest that impression formation is less positive towards children with autism spectrum disorder than to typically developing children.
\end{abstract}

Keywords: autism spectrum disorder, facial expressivity, first impressions, friendship formation 


\section{Does facial expressivity count? How typically developing children respond initially to children with autism.}

Autism Spectrum Disorder (ASD) is a developmental disorder characterised by impairments in social interaction, verbal and non-verbal communication and repetitive and stereotyped behaviours (American Psychiatric Association, 2000). As part of the social and communication deficits, individuals encounter difficulties making affective contact with others. This may include problems with understanding other peoples' facial expressions (Tardif et al., 2007; Lindner and Rosén, 2006; Weeks and Hobson, 1987) or difficulties with interpreting the intentions of others (Spek et al., 2010; Baron-Cohen, 1995). Atypical facial expressivity, and a predominant display of negative emotion, over represented in individuals with ASD (Bieberich and Morgan, 1998; Capps et al., 1993). Lack of expressivity may hamper social discourse or provoke negative initial reactions to the person with ASD. Despite research on emotional expressivity in ASD, little is still known about the influence of general facial expressivity on the formation of first impressions. Children with ASD are increasingly integrated into mainstream education (Ellis et al., 2008) where they are often faced with the negative responses of others (Iobst et al., 2009; Swaim and Morgan, 2001). It is therefore particularly important to understand the factors that may 
influences impression formation. The small-scale study reported in this paper investigated non-verbal communication from an interpersonal, rather than a dispositional, approach. Our emphasis was on how typically developing (TD) children initially respond to those with ASD, with specific focus on the influence of the facial expressivity of the ASD individual. Studies that have addressed facial expressivity in ASD uphold Kanner’s (1943) initial observation of flat affect or odd facial expressivity within this population. Flat affect appears to be a feature of autism rather than a general feature of developmental delay (Yirmiya et al., 1989; Snow et al., 1987). For example, Loveland, Tunali-Kotoski, Pearson and Brelsford (1994) reported that participants with ASD produced fewer recognisable facial expressions and significantly more 'bizarre' and 'mechanical' facial expressions than a Down's Syndrome comparison group. Their study used posed rather than naturally occurring expressions. In play situations, designed to elicit spontaneous facial expressions, children with ASD may have difficulty coordinating their expressions to fit the needs of shared social interaction (Dawson et al., 1990). They may also respond inappropriately to situational cues (Reddy et al., 2002). Whilst emotional displays may be apparent, parents report that their children tend to exhibit more negative displays rather than positive displays such as smiling (Capps et al., 1993; Bieberich and Morgan, 1998). The focus of our study was on the initial impressions a TD child may form of an ASD child even before social 
interaction is initiated. Problems with facial expressivity could negatively affect the first impressions made by individuals with ASD.

Impressions of others are rapidly formed (Willis and Todorov, 2006; Bar et al., 2006), can be persistent and influence subsequent behaviour (Hendrick and Costantini, 1970; Stewart, 1965). The duration for which a face is displayed, in experimental studies, does not change the impression initially formed by the viewer. For example, judgments about an individual's personality traits have been shown to be consistent whether the participant is viewing a video for 30 seconds or 4-5 minutes (Ambady and Rosenthal, 1992). Judgments made of others are based upon a variety of factors including: appearance, physical behaviours, speech and non-verbal communication (Palmer and Simmons, 1995; Butler et al., 2003; Todorov and Uleman, 2003). These factors are used to infer traits such as trustworthiness, competence, aggression, helpfulness and attractiveness, and contribute to first impressions of a person's likeability (Willis and Todorov, 2006). If a TD child's initial impression of an ASD child is negative this may influence subsequent friendship formation.

We wished to gauge the initial reactions of TD children towards children with ASD and examine whether possible negative reactions may be related to facial expressivity. In order to do this, we asked TD children and adults to rate silent videos of ASD and TD 
children. We investigated two main hypotheses. The first hypothesis predicted that the TD children in the videos would be rated as having more expressive faces than the children with ASD. Further, we anticipated that reduced expressivity would result in lower friendship ratings; therefore, the second hypothesis predicted that friendship ratings would be higher for the TD than the ASD Children in the videos. It is hoped that the findings will increase understanding of factors that may influence the formation of friendships between children with ASD and their TD peers.

Method

Design

The study elicited subjective ratings from adults to short video clips of children. The ratings were given for attractiveness and expressivity. These were analysed using sa repaeated measures ANOVA. The videos were subsequently rated by child participants using a questionnaire to measure impression formation. These ratings were analysed by paired sample t-tests with Bonferroni corrections applied. 


\section{Participants}

Experiment 1. Adult participants were recruited to assess the expressivity of the children in the videos. Participants $(n=25)$ were aged between $20-70$ years (mean age $=31$ years, $\mathrm{SD}=16.34$ ) and were recruited from staff and students at xxxxxxxxxxxx. The participants were naïve to the goal of the study and were not drawn from staff or students working in the psychology department. From these participants, one was working in a professional capacity with children, one participant had children and two participants had grandchildren of similar ages to the children in the study Experiment 2. The participants $(n=44)$ were mainstream pupils from a XXXXX Education Authority Primary School, aged between $10-11$ years (mean age $=10.37$ years, SD $=0.34$ ). Parents were fully informed of the study through individual letters. The school adopted an opt-out policy, which asked parent to notify the school if they did not want their children to take part in the study. Two children from the year group opted out of the study.

Ethical approval for the study was granted by the department of psychology ethics committee at XXXXXXXXXXXXXXX. 


\section{Materials}

Stimuli were produced using eight participants. The participants comprised of two girls and two boys with ASD (mean age: 11 years 6 months, range: 10 years 5 months -12 years 3 months) and two typically developing boys and two girls (mean age: 12 years 2 months, range: 11 years 6 months-12 years 8 months). The ASD children had been diagnosed by a clinician, and they were attending a school for children with autism. These children were selected by a member of staff who was not involved in the study and had no knowledge of the intention of the study. The criteria for selection were that the child should be considered academically capable and were considered capable of talking about themselves, their interests and their family. Two of the children originally involved in the study have since joined mainstream state schools. The TD children were recruited from colleagues of the researchers. For simplicity, the participants taking part in the videos will be referred to as actors. The actors were filmed for approximately 10 minutes while talking about their daily lives, families and interests. All of the children gave fluent answers to the questions. The interviews were all conducted by the same researcher. The researcher sat across the table from them and asked each child an identical set of questions in the same order (see Appendix A). For reasons relating to ethics, the questions were designed to elicit 
positive emotional responses. All actors knew they were being filmed. They did not know the study was about facial expressivity, but they knew the study was related to friendship.

The final video clips were edited to produce short 50-second videos. Ten second segments were edited together to produce the final clip. Each ten-second segment was taken at consecutive one-minute intervals starting one-minute after the interview had commenced until five ten second clips had been taken. The sound track was removed to focus participants' attention upon the actor's facial expressions and reduce any influence of variability in language skills between the actors.

Measures

Questionnaires were created by adapting factors previously employed in research investigating first impression formation (Willis \& Todorov, 2006; Boutot, 2007). The wording was kept as simple and direct as possible (see Appendix B). The questions were piloted on a group of ten-year-old pupils prior to the study, and changes were made based on their comments. Seven questions measured the actor's trustworthiness, kindness, competence, similarity to the rater, attractiveness and the rater's desire to play with and befriend the actors. The key question "Would you want to be friends with him/her" was included as question three, to avoid first or last question bias. Internal consistency was 
measured resulting in a Cronbach’s alpha of .79. All the questions were rated on a bipolar scale (E.g. kind/ unkind). In order that ratings could be converted into continuous data, raters made their responses by bisecting a line that ran underneath each question. Each line was equal in length and consisted of two anchor points. For example, for the question 'Do you think he/she would be unkind to you?', the line was anchored at never and always. Data were recorded as the number of centimetres the bisection occurred away from the furthermost left of the line. Therefore, for the question relating to kindness a score of $0 \mathrm{~cm}$ would indicate never unkind, and a score of $8 \mathrm{~cm}$ would indicate always unkind. The adult participants responded in an identical manner but were only asked to rate attractiveness and expressivity.

\section{Procedure}

The child participants rated the video presentations using the devised rating scale.

Understanding of the task was assessed through the completion of two practice trials. The same procedure was used for the adult participants.

Participants rated the videos while watching each actor. This was done by the participant marking a point on the lines under each of the friendship questions. A trial set of questions was given to each participant before the study commenced. The participant 
rated a still photo of a child not used in the study. The participant discussed their choices in order for the researcher to judge the participant's level of understanding of the task. Videos were presented in a randomised order. The participants were informed that their answers were anonymous. The researcher sat with her back to the participant during the completion of the questionnaire. After each of the video presentations, the recording was paused on a blank screen until the participant indicated that they were ready to continue. 


\section{Results}

\section{Experiment 1}

Experiment 1 tested the hypothesis that the actors with ASD would be rated as less facially expressive than would the TD actors. Adult participants provided the ratings. Mean scores for both TD and ASD children are shown in Table 1.

\section{[PLACE TABLE 1 AROUND HERE]}

A repeated measures ANOVA revealed that the ASD actors were rated as less expressive $(M=3.52)$ than the TD actors $(M=4.83), F(1,24)=9.42, p=.005, d=1.24$. The ASD actors were also rated as less attractive $(M=4.30)$ than the TD actors $(M=4.82)$, $F(1,24)=32,30, p<.001, d=2.30$. There was also a significant Expressivity $\mathrm{x}$ Attractiveness interaction $F(1,24)=13.39, p<.001, \mathrm{~d}=1.47$. The interaction effect was caused by a significant disparity in attractiveness and expressivity ratings for the ASD actors. Post hoc test revealed no significant difference between these two variables when data from the TD group were analysed $t(24)=.05, p=.96$. For the ASD group expressivity ratings $(M=3.52)$ were significantly lower than attractiveness ratings $(M=4.30)$, $t(24)=4.34, p=<.01, d=.78$. The results suggest that our 25 raters differentiated between 
the ASD and TD actors based on expressivity and attractiveness. Importantly whilst attractiveness and expressivity rating were identical for the TD group expressivity rating for the ASD group were lower than their attractiveness ratings. This suggests that the expressivity ratings for this group are not simply attractiveness by another name.

\section{Experiment 2}

Experiment 2 tested the hypotheses that friendship ratings would be higher for TD than for the ASD actors. Table 2 shows the mean scores given to the ASD and TD actors for the friendship component variables. Paired sample t-tests were used to assess the ratings, and alpha values, after applying Bonferroni corrections for multiple tests, are given in Table 2.

\section{[PLACE TABLE 2 AROUND HERE]}

The results demonstrated that ratings for the specific friendship measure (question 3) were significantly higher for the $\mathrm{TD}$ actors $(\mathrm{M}=4.67, \mathrm{SD}=1.21$ ) compared to the ASD actors $(M=4.0, S D=1.16)$. Similarly, the TD actors were rated statistically significantly higher on all the variables used to measure aspects of friendship. The results 
Running head: Facial expressivity and friendship formation.

suggest that even when blind to the nature of the study and to the diagnosis of the actors in the videos, the ratings given by TD children differentiated the TD and ASD actors. 


\section{Discussion}

The primary question addressed in the present study was whether friendship appraisals would be higher for videos of typically developing children compared to those for children with an Autism Spectrum Disorder. The results supported our hypotheses. Across all of the friendship measures, the actors with ASD were rated lower than the TD actors. Importantly, the children making friendship judgements were unaware that they were rating both TD and ASD children. The TD actors in the videos were also rated by adults as having more expressive faces than the ASD actors. Both of these sets of results reflect the subjective views of the raters. Taken together, these findings suggest that the lack of expressivity of the ASD actors, in this study, influenced the TD participants' first impressions of them. This was true even though there was no social interaction between the raters and the actors.

The study reported in this paper demonstrated that expressivity is associated with friendship ratings, even when exposure is brief. Our method elicited spontaneous facial expressions from the actors in our videos. This would suggest that some children with autism have difficulty producing spontaneous facial representation of mood and emotion to the same quality or extent as TD children. Currently, it is unknown exactly why facial 
expressivity influences friendship appraisals; an inexpressive face may afford the viewer minimal information about the emotional state, and behavioural intentions of the agent (Argyle, 1994; Ekman et al., 1987). In our study, typically developing individuals may have found it hard to appraise the intentions of the actors with ASD. In face-to-face communication, these effects would be further compounded (Bieberich and Morgan, 1998; Capps et al., 1993). Our study demonstrated that even when the rater was not involved in the interaction, and the scene was focused solely on the ASD actor, the ASD actor was still rated lower than TD actors on the friendship measures.

The current study has theoretical implications for the developmental trajectory of ASD. Hobson (1993) and more recently Klin, Jones, Schultz, and Volkmar (2005) have suggested that the social impairments in ASD may be partly explained in terms of a lack of experiences of reciprocal emotional relatedness with others. These theories suggest that the ability to express oneself non-verbally is a prerequisite for the development of an understanding of others' minds. In this respect, individuals with ASD may be delayed in developing an adequate concept of mind because of an insufficient quantity and quality of experiences surrounding shared emotional expressivity with others. Whilst theory of mind is an ability which can be seen in young children it is now thought that it is a skills which improves throughout development and into adulthood and is best describes on a continuum 
from poor to good rather than as a binary absent or present (Apperly et al., 2009; Samson et al., 2010). Poor facial expressivity may further remove individuals with ASD from meaningful interactions. In our study, quality of facial expressivity negatively affected the immediate judgements made by others, and this may impede children from approaching ASD children in a school setting. The TD children in our study were less likely to rate the ASD actors, in comparison with the TD actors, as someone they would want to befriend. Further, our correlational analysis suggested these findings might generalise to the lives of some children with ASD.

The small number of children used as actors in this study obviously limits the findings. Although the ASD actors were fluent in their responses and gave information freely, we cannot conclude that they are representative of a population that is known to be heterogeneous in nature. The ASD actors were nevertheless articulate and willing to talk about their experiences. The results do provide an initial indication that the ASD actors, when viewed briefly and in situations in which dyadic interaction was not important, were still rated as less expressive and less likable than were the TD actors. It is noted that the interviews conducted with the children revolved around social situations. Whilst this may be similar to topics children are likely to talk about in schools (e.g. holidays, friends, family) these types of questions may not have elicited a full range of expressive responses 
from the children with ASD. Subjects such as favourite hobbies or specific idiosyncratic pleasures may have elicited a different response and this is something further studies will seek to explore.

The findings of the present study have value in the applied setting of schooling. Children with ASD experience more peer rejection and have fewer friendships than their TD peers (Church et al., 2000; Chamberlain et al., 2007). Negative peer responses can be especially upsetting for more socially aware children with ASD who may be striving and failing to form friendships (Whitehouse et al., 2009; Kasari and Rotheram-Fuller, 2007).We suggest that this division can begin from minimal contact between typically developing and ASD children . Improving social cohesion between these two groups is important for two main reasons. First, an increase in the quality of social relationships between ASD and TD peers can have a major influence on the social and academic development of both parties (Wentzel et al., 2004). Second, improving social cohesion for those with ASD in a mainstream setting may decrease levels of anxiety in these children (White and RobersonNay, 2009). Some studies have suggested that children with ASD can be taught to display appropriate affective facial expressions (Gena et al., 1996): however these studies are generally evaluated within highly restricted environments and often assess the ability of the ASD child to produce an emotion rather than the impact the training on the ASD child's 
peer group. We suggest an interpersonal as opposed to dispositional approach to helping ASD individuals adjust to mainstream schools. Interventions aiming to improve TD children's attitudes towards their ASD peers may be more effective then attempting to improve the facial expressivity of the ASD individuals themselves. For example, remediation schemas such as the Children Friendship Training which promotes inter-social skills in both TD and ASD children, has proven effective in promoting friendship between these groups (Frankel et al., 2010; Frankel and Whitham, 2010).

\section{Conclusion}

Facial expressivity plays an important role in building relationships and influences first impression judgments about likeability. Preliminary findings from our small-scale study suggest that these judgments are negatively influenced in TD children by ASD children's reduced facial expressivity. We have suggested that addressing negative bias against choosing a child with ASD as a potential friend requires a both an intra- and interpersonal approach with intervention focusing on both the ASD and TD child. 


\section{References}

Ambady N and Rosenthal R. (1992) Thin slices of expressive behaviour as predictors of interpersonal consequences: a meta-analysis. Psychological Bulletin 111: 256-274. American Psychiatric Association. (2000) American Psychiatric Association. Diagnostic and Statistical Manual of Mental Disorders, Washington, DC.

Apperly IA, Samson D and Humphreys GW. (2009) Studies of adults can inform accounts of theory of mind development. Developmental Psychology 45: 190-201.

Argyle M. (1994) The psychology of interpersonal behaviour, London: penguin.

Bar M, Neta M and Linz H. (2006) Very first impressions. Emotion 6: 269-278.

Baron-Cohen S. (1995) Mindblindness: An essay on autism and theory of mind, Cambridge, MA: MIT Press/Bradford Books.

Bieberich AA and Morgan SB. (1998) Affective expression in children with autism or Down syndrome. Journal of Autism and Developmental Disorders 28: 333-338.

Butler EA, Egloff B, Wilhelm FH, et al. (2003) The social consequences of expressive suppression. Emotion 3: 48-67.

Capps L, Kasari C, Yirmiya N, et al. (1993) Parental perception of emotional expressiveness in children with autism. Journal of Consulting and Clinical Psychology 61: 475-484.

Chamberlain B, Kasari C and Rotheram-Fuller E. (2007) Involvement or isolation? The social networks of children with autism in regular classrooms. Journal of Autism and Developmental Disorders 37: 230-242.

Church C, Alisanski S and Amanullah S. (2000) The social, behavioral, and academic experiences of children with Asperger syndrome. Focus on Autism and Other Developmental Disabilities 15: 12-20. 
Dawson G, Hill D, Spencer A, et al. (1990) Affective exchanges between young autistic children and their mothers. Journal of Abnormal Child Psychology 18: 335-345.

Ekman P, Friesen WV, O'Sullivan M, et al. (1987) Universals and cultural differences in the judgments of facial expressions of emotion. Journal of Personality and Social Psychology 53: 712-717.

Ellis S, Tod J and Graham-Matheson L. (2008) Research report: special educational needs and inclusion, reflection \& renewal. Birmingham: NASUWT.

Frankel F, Myatt R, Sugar C, et al. (2010) A randomized controlled study of parent-assisted children's friendship training with children having autism spectrum disorders. Journal of Autism and Developmental Disorders 40: 827-842.

Frankel F and Whitham C. (2010) Parent-assisted group treatment for friendship problems of children with autism spectrum disorders. Brain Research.

Gena A, Krantz PJ, McClannahan LE, et al. (1996) Training and generalization of affective behavior displayed by youth with autism. Journal of Applied Behavior Analysis 29: 291304.

Hendrick C and Costantini AF. (1970) Effects of varying trait inconsistency and response requirements on the primacy effect in impression formation. Journal of Personality and Social Psychology 15: 158-164.

Hobson P. (1993) Understanding Persons: The Role of Affect. In: Cohen SB, Flusberg HT and Cohen DJ (eds) Understanding Other Minds: Perspectives From Autism New York: Oxford Press, 204-227.

Iobst E, Nabors L, Rosenzweig K, et al. (2009) Adults’ perceptions of a child with Autism. Research in Autism Spectrum Disorders 3: 401-408. 
Kanner L. (1943) Autistic disturbances of affective contact. Nervous Child 2: 217-250.

Kasari C and Rotheram-Fuller E. (2007) Peer Relationships of children with autism: Challenges and interventions. In: Hollander E and Anagnostou E (eds) Clinical manual for the treatment of autism. Arlington, VA US: American Psychiatric Publishing, Inc., 235-257.

Klin A, Jones W, Schultz RT, et al. (2005) The Enactive Mind-From Actions to Cognition: Lessons from Autism. Hoboken, NJ,US: John Wiley \& Sons, Inc, xxv, 703.

Lindner JL and Rosén LA. (2006) Decoding of Emotion through Facial Expression, Prosody and Verbal Content in Children and Adolescents with Asperger's Syndrome. Journal of Autism and Developmental Disorders 36: 769-777.

Loveland KA, Tunali-Kotoski B, Pearson DA, et al. (1994) Imitation and expression of facial affect in autism. Development and Psychopathology 6: 433-444.

Palmer MT and Simmons KB. (1995) Communicating intentions through nonverbal behaviors conscious and nonconscious encoding of liking. Human Communication Research 22: $128-160$.

Reddy V, Williams E and Vaughan A. (2002) Sharing humour and laughter in autism and Down's syndrome. British Journal of Psychology 93: 219-242.

Samson D, Apperly IA, Braithwaite JJ, et al. (2010) Seeing it their way: Evidence for rapid and involuntary computation of what other people see. Journal of Experimental Psychology: Human Perception and Performance 36: 1255-1266.

Snow ME, Hertzig ME and Shapiro T. (1987) Expression of emotion in young autistic children. Journal of the American Academy of Child \& Adolescent Psychiatry 26: 836-838.

Spek AA, Scholte EM and Van Berckelaer-Onnes IA. (2010) Theory of mind in adults with HFA and Asperger syndrome. Journal of Autism and Developmental Disorders 40: 280-289. 
Stewart RH. (1965) Effect of continuous responding on the order effect in personality impression formation. Journal of Personality and Social Psychology 1: 161-165.

Swaim KF and Morgan SB. (2001) Children's attitudes and behavioral intentions toward a peer with autistic behaviors: Does a brief educational intervention have an effect? Journal of Autism and Developmental Disorders 31: 195-205.

Tardif C, Lainé F, Rodriguez M, et al. (2007) Slowing down presentation of facial movements and vocal sounds enhances facial expression recognition and induces facial-vocal imitation in children with autism. Journal of Autism and Developmental Disorders 37: 1469-1484.

Todorov A and Uleman JS. (2003) The efficiency of binding spontaneous trait inferences to actors’ faces. Journal of Experimental Social Psychology 39: 549-562.

Weeks SJ and Hobson RP. (1987) The salience of facial expression for autistic children. Journal of Child Psychology and Psychiatry 28: 137-151.

Wentzel KR, Barry CM and Caldwell KA. (2004) Friendships in Middle School: Influences on Motivation and School Adjustment. Journal of Educational Psychology 96: 195-203.

White SW and Roberson-Nay R. (2009) Anxiety, social deficits, and loneliness in youth with autism spectrum disorders. Journal of Autism and Developmental Disorders 39: 10061013.

Whitehouse AJO, Durkin K, Jaquet E, et al. (2009) Friendship, loneliness and depression in adolescents with Asperger's syndrome. Journal of Adolescence 32: 309-322.

Willis J and Todorov A. (2006) First Impressions: Making Up Your Mind After a 100-Ms Exposure to a Face. Psychological Science 17: 592-598. 
Yirmiya N, Kasari C, Sigman M, et al. (1989) Facial expressions of affect in autistic, mentally retarded and normal children. Journal of Child Psychology and Psychiatry 30: 725-735. 


\section{Appendices}

Appendix A

Interview Questions

What is your name?

How old are you?

What class are you in?

Who is your teacher?

Do you like her?

Tell me about her

Who is your best friend in school?

Why do you like them?

Have they ever done something to make you laugh?

Tell me about it.

Who is in your family?

Who do you like best in your family?

Why do you like them?

What is your best thing that you do with them?

Tell me about it

What about your brothers/ sisters?

Do they make you laugh?

Tell me about him/ her/ them

Have you been away to the caravan/ on holiday/ on any special outings this year?

Did you have a happy time?

What did you like best?

Tell me about it.

Last few questions now -

Do you have a special hobby or something you like best in the whole world - toy, game, book, film?

Why do you like it?

Tell me about it.

OK questions are finished now.

Do you have any questions for us?

Thank you for helping us. 
Running head: Facial expressivity and friendship formation.

Appendix B

Questions asked when children rated the videos

1. Would you tell him/her a secret?

2. Would you play with him/her?

3. Would you want to be friends with him/her?

4. Do you think this person could help you with a maths problem?

5. Would he/she be unkind to you?

6. Do you think they would like the same things as you? 
Running head: Facial expressivity and friendship formation.

\section{Footnotes}

[Insert footnotes here, do not use Word's footnoting function] 
Table 1

Adult ratings for expressivity and attractiveness

ASD $\quad$ TD

Expressivity $\quad 3.52(0.93) \quad 4.83(0.80)$

Attractiveness $4.30(1.05) \quad 4.83(0.90)$

Table 2

Children's ratings on the friendships measures and effect size reported as Cohen's $d$

\begin{tabular}{lllll}
\hline & ASD & TD & $d$ \\
\hline Friendship & $4.00(1.16)$ & $4.67(1.21) * *$ & 0.56 \\
Trustworthiness & $2.98(1.30)$ & $3.79(1.36) * *$ & 0.61 \\
Play & $3.86(1.21)$ & $4.48(1.17) * *$ & 0.52 \\
Helpfulness & $4.46(1.15)$ & $4.86(1.24) *$ & 0.33 \\
Similarity & $2.96(1.01)$ & $3.42(1.13) * *$ & 0.43 \\
Attractiveness & $3.71(1.09)$ & $4.44(1.05) * *$ & 0.70 \\
Composite score & $3.65(0.95)$ & $4.25(0.91) * *$ & 0.65 \\
\hline${ }^{*}<<.01,{ }^{* *}<.001$ & & & &
\end{tabular}


Running head: Facial expressivity and friendship formation.

Figure Captions

[Insert Figure Legends here] 
[First Authors Last Name] Page 30

1 [Insert Figures here] 